\title{
Similarities in Asymptomatic HIV Infection and Cancer: A Common "Driver" Hypothesis
}

\author{
Mburu Samuel \\ School of Health Sciences, Kirinyaga University, Kenya
}

Copyright $(2017$ by authors, all rights reserved. Authors agree that this article remains permanently open access under the terms of the Creative Commons Attribution License 4.0 International License

\begin{abstract}
Cancer and Human Immunodeficiency Virus/Acquired Immunodeficiency Syndrome (HIV/AIDS) are associated with chronic oxidative stress, inflammation and immune activation either as a consequence or a cause. Despite well documented characteristic similarities in the two diseases, this has not been exploited for better understanding of carcinogenesis. The purpose of this descriptive study was to review existing studies for knowledge, research gaps in the role of oxidative stress, inflammation, immune activation in cancer and asymptomatic HIV infection; identify similarities, differences to stimulate new research ideas which can accelerate future therapeutic target discoveries. PubMed, ScienceDirect and Google scholar databases were searched using the keywords: oxidative stress, inflammation, immune activation, cancer and asymptomatic HIV infection. Little research has been done on immune evasion, tolerance and oxidative stress-induced inflammation and immune activation as therapeutic targets in both diseases. Senescence and the role of respiratory burst in HIV infection have not been exhaustively studied. Out of a total of 15,788,387 hits, $15,284,572$ hits related to similarities with only 503, 815 relating to the differences between the two diseases. Consequently and after pearling, 89 articles that were directly relevant to the study were selected. After critical appraisal, the identified studies were analyzed, results compared and presented in form of summary tables. Results indicated that chronic oxidative stress, inflammation and immune activation were common drivers of progression in the two diseases. Therefore, better understanding of these drivers might provide new mechanistic insights in carcinogenesis and provide future novel therapeutic targets. This will support the United Nations sustainable development goal (SDG) number 3 on ensuring health lives and promoting well-being for all at all ages.
\end{abstract}

Keywords Oxidative Stress, Inflammation, Immune Activation, Cancer, Asymptomatic HIV Infection

\section{Introduction}

The emergence of non-communicable diseases (NCDs) such as cancers has added to HIV/AIDS burden especially in resource-limited settings in the sub-Saharan Africa [1, 2]. Annually, approximately 40,000 Kenyans are diagnosed with cancer, of which, about 27,000 dies within 1-2 years of diagnosis [3]. Likewise, about 80,000 annual HIV/AIDSrelated deaths and 100,000 new HIV infections occurred in Kenya in 2012 [4]. This makes the two diseases the leading causes of mortality in Kenya [5]. Importantly, with the expected prolonged life of people living with HIV/AIDS due to universal access to antiretroviral therapy, HIV-related malignancies are expected to increase 200 fold, aggravating an already dire situation [6].

Cancer and HIV/AIDS are associated with chronic oxidative stress, inflammation and immune activation, though degree varies, hence similarities and differences in their effects in the two diseases [7, 8]. For that reason, understanding, drivers of these similarities and differences, their impact in the two diseases could be exploited for better understanding of carcinogenesis, cancer progression and maintenance. Regrettably, there is no data currently on studies relating the two diseases, despite existing of extensive, data on the independent diseases.

In addition, to stimulating more research interest in carcinogenesis, providing future novel therapeutic targets, providing preventive, screening, early diagnosis strategies and policies, such studies will have a direct impact on cancer management.

\section{Current Knowledge in Carcinogenesis}

The influence of free radicals, proto-oncogenes, oncogenes on initiation, promotion and evolution towards malignancy has been known from as early as 1990 [9]. Significantly, oxidative stress has been recognized as a potent initiator of the consistent NF-kB activation strongly associated with chemical tumorigenesis, tobacco, alcohol, high-fat diet, environmental pollutants, cancer-inducing viruses (HIV, HPV, Hepatitis B,C), bacteria ( $H$. pyroli), ultraviolet light, ionization radiation and obesity-associated 
cancers [10,11]. Furthermore, oxidative stress has been recognized as the "master key" through which tumour microenvironment interacts with almost all cancer hallmarks [12].

In cancers, persistent but moderate viral, bacterial or other carcinogens-induced oxidative stress, inflammation and immune activation leads to formation of lesions eliciting a vicious cycle of the same and persistent nuclear factor of Kappa B (NF-kB) signaling [13,14]. Though persistent, the NF-kB signaling is not strong enough to lead to apoptotic signaling, this together with cellular adaptations to reactive oxygen species (ROS), such as change to replicative immortality signaling by antioxidant defenses enables resistance to cell death as a defense mechanism [15].

ROS and oxidative stress's capacity to induce metabolic reprogramming [14], stimulate proliferative signaling [16, 17], activate angiogenic signaling [18], influence cellular senescence [19] as well as induction of deoxynucleic acid (DNA) damage [17] has been reported previously. Furthermore, majority of cancers, especially solid tumours, myeloproliferative disorders commence in a setting of oxidative stress and inflammation [20, 21]. Importantly, chronic inflammation, though commonly caused by infections, can also be produced by exposure to carcinogens such as irradiation and environmental chemicals [22, 23].

In cancers, oxidative stress and glycolytic influx is thought to lead to metabolic reprogramming [24], while increased respiratory burst in asymptomatic HIV infection has been reported [25,26,27,28]. In addition, chronic oxidative stress impacts on the immune status of the host by activating the innate and adaptive cells, thereby compromising immunosurveillance [29].

Consequently, the host's immunity plays a critical role in determining cancer initiation and its progression [30]. Interestingly, immunosuppression has been recognized as the biggest risk factor for solid tumours such as non-Hodgkin lymphoma [31]. Essentially, cancer is associated with increased immune activation of both innate and adaptive immune systems, characterized by expression of tumour antigens, cytokines production (Interleukin 8 [IL-8], transforming growth factor beta [TGF- $\beta$ ], tumour necrosis factor alpha [TNF- $\alpha$ ], IL-I and IL-6), induction of immunosuppressive FOXP3+ T-regulatory cells (T-regs), down regulation of major histocompatibility complex I (MHC I) molecules on cytotoxic T-cells (CD8+ T-cells) [32,33], dysfunctional CD8+ T-Cells, all believed to be driven by oxidative stress and or inflammation [34, 35,36]. In addition, T-regs have been shown to be essential for K-Ras-mediated lung tumorigenesis [37]. A "bystander effect" produced by ROS and nitrogen oxide (NO) radicals from activated macrophages are thought to contribute to genomic instability and leukomogenesis [39].

Cancer development and progression is characterized by the following seven hall marks [33, 34];

1. sustained proliferative signaling

2. evading growth suppression

3. activating tumour invasion and metastasis
4. enabling replicative immortality

5. inducing angiogenesis

6. resisting cell death

7. immune evasion and tolerance

\section{Current Knowledge in Asymptomatic HIV Infection}

Depending on the severity of symptoms, world health organization (WHO) classifies HIV infection into four clinical stages; clinical stage 1 or asymptomatic stage, which has no symptoms or characterized by general lymphadenopathy, clinical stage 2 with mild symptoms, clinical stage 3 with advanced symptoms and clinical stage 4 or the acute AIDS phase with severe symptoms such as the HIV wasting syndrome among others [39]. The hallmark of HIV infection is the massive depletion of CD4+ T-helper cells and the subsequent inability to fight opportunistic infections [40]. This occurs during the early phases of HIV infection, more specifically, stage II of HIV infection and has been associated with increased free radicals (ROS) generation [41, 42, 43, 44]. Similarly, some viral proteins (such as Vpr) are capable of binding to mitochondria of infected CD4+ T-cells, directly inducing apoptosis [45]. Hence, asymptomatic HIV infection is characterized by chronic oxidative stress, inflammation and immune activation, which are associated with the massive depletion of CD4 T-Cells [13, 46].

Oxidative stress has been defined as the cytopathological consequence of an imbalance between free radical production and the antioxidant status of the cell by Franco \& Panayiotadis, (2009) [47]. This is believed to contribute considerably to the death of CD4+ T-lymphocytes in HIV infection, thereby aiding progression to AIDS [48, 49, 50]. Further, the sensitivity of immune cells to oxidative stress due to high polyunsaturated acyl lipids in their plasma membrane makes them vulnerable to peroxidation. As a result, their biomolecules such as DNA, carbohydrates, protein and uric acid are prone to oxidative damage by ROS $[51,52]$. Specifically, hydrogen peroxide $\left(\mathrm{H}_{2} \mathrm{O}_{2}\right)$ and other oxidants generated by activated neutrophils, macrophages in HIV infection during respiratory burst (due to increased energy demands), adenosine triphosphate (ATP) production, during inflammation [54], promotes tissue injury and inflammation [55, 56]. As a result, respiratory burst, which has been reported in asymptomatic HIV infection, is believed to contribute to the overall oxidative stress and the associated massive death of CD4+ T-cells in early HIV infection [27, 56].

Increased ROS and oxidative stress in HIV infection, leads to persistent NF-kB signaling, which ultimately causes apoptosis (programmed cell death) of CD4 T-lymphocytes. Likewise, translocation of microbial products, such as lipopolysaccharides (LPS) across the gastrointestinal tract (GIT) mucosal lining during early HIV infection adds to the persistent immune activation and inflammation [45, 57, 58]. Consequently, the "leaky gut" phenomenon has been identified as an essential source of the characteristic persistent immune stimulation in HIV infection [59, 60]. 
Accordingly, this results in oxidative stress, dysfunctional immune system, pro-inflammatory cytokine production, inflammation-associated complications and uncontrolled viral replication in activated CD4+ T-cells [56, 61]. For that reason, increased immune activation, inflammation and oxidative stress have been linked to death of immune cells, rapid disease progression and a higher risk of death [59, 62, $63,64]$.

In addition, early HIV infection is characterized by immune evasion and tolerance through down regulation of MHC I molecules on CD8 T-lymphocytes. As a result, CD8 T-cells becomes dysfunctional, cannot recognize or destroy virally-infected cells, thereby enabling immune evasion and tolerance common in HIV/AIDS. Recently, Nolan et al. (2016) [65] reported that T-cell activation levels expressed as CD38 on CD8+ T-cells, predicted a poor prognosis for the HIV infected patients, which has been confirmed by other investigators [59, 66, 67]. The persistent systemic immune activation in HIV infection results in increased pro-inflammatory cytokines generation such as TNF- $\alpha$, IL-I and 6, which have been implicated in the activation of extrinsic pathway of apoptosis [57,68]. Subsequently, the generated pro-inflammatory cytokines drive several processes underlying the inflammatory response, cell apoptosis, activation and differentiation [69, 70]. As a result, markers of inflammation, immune activation have been recognized as stronger predictors of HIV progression to AIDS than CD4 count and viral load [71].

\section{Objectives}

To investigate the hypothesis that oxidative stress, inflammation and immune activation drives the characteristic similarities in pathophysiology of cancer, early HIV infection, the author reviewed existing literature to identify critical knowledge gaps, unmet research needs specifically on interrelations between oxidative stress, inflammation, immune activation in relation to the two diseases.

The objectives of this descriptive review were to:

1. Review existing literature for similarities, differences in pathophysiology of cancer and asymptomatic HIV infection,

2. Identify knowledge, unmet research gaps in order to stimulate further research interest in the field.

3. Exploit the identified knowledge, research gaps to generate new hypothesis, new mechanistic insights in carcinogenesis, tumour maintenance, progression in order to accelerate discovery of new therapeutic targets and preventive strategies.

The author was trying to answer the following review questions whose answers hold the key to understanding carcinogenesis, cancer maintenance and its progression:

1. At what point does apoptotic and or NF-kB signaling common in cancer and HIV shift to sustained proliferative signaling/replicative immortality in cancer and why?
2. What drives the shift in apoptotic signaling; i.e. from cell death of CD4-T-lymphocytes in asymptomatic HIV infection to resistance to cell death (apoptosis)/proliferative signaling/replicative immortality in cancer?

3. What causes metabolic reprogramming in cancer and only an increased respiratory burst in HIV?

4. What causes cellular senescence in HIV/AIDS and why is it absent in cancer?

5. What causes clonal selection in cancers and why is it absent in HIV?

6. What is the impact of oxidative stress, inflammation and immune activation in cancer and asymptomatic HIV infection?

7. What is the role of immune system in progression of cancer and HIV/AIDS?

\section{Materials and Methods}

This was a descriptive review of the existing literature on cancer, asymptomatic HIV infection, their similarities and differences in pathophysiology. This study was conducted at Kirinyaga University from $5^{\text {th }}$ March 2016 to $27^{\text {th }}$ February 2017. The inclusion criteria was only full text articles in English, publications on processes, mechanisms in early HIV infection, oxidative stress, antioxidants, metabolic reprogramming, respiratory burst, hypoxia, apoptosis, inflammation, immune activation, major histocompatibility molecules, immune evasion, tolerance, senescence, mutations, clonal selection, therapeutic targets in cancer and asymptomatic HIV infection. The exclusion criteria was all publication not in English language, not directly associated with the search words; asymptomatic HIV infection, cancer, oxidative stress, inflammation, immune activation, publications involving all the other diseases apart from cancer and asymptomatic HIV infection.

The keywords were developed by breaking down the review questions. From the objectives and review questions, a search strategy using different combinations of the key search words in relation to either asymptomatic HIV infection or cancer was devised and applied. The following databases; PubMed, Google scholar and ScienceDirect were searched and downloaded at Kirinyaga University from $6^{\text {th }}$ of April, 2016 to $30^{\text {th }}$ November, 2016, using the previously identified keywords. A total of 15,788,387 hits for both similarities and differences between the two diseases were obtained. After pearling, critical appraisal of the studies to assess the relevance to the current study, quality, what was analyzed, results obtained of the downloaded articles, 89 articles were selected. The articles were reviewed and the relevant background information, current knowledge in important similarities, differences between the two diseases, research gaps in the field identified, highlighted, compared and documented. The results of the relevant articles were compared, appraised, analyzed, summarized and presented in the form of summary tables. 
Table 1. Existing studies related to the identified similarities in cancer and asymptomatic HIV infection

\begin{tabular}{|c|c|c|c|c|c|c|c|c|c|}
\hline Databases/Cancer & $\begin{array}{l}\uparrow \text { Oxidative } \\
\text { stress }\end{array}$ & $\downarrow$ Antioxidant & $\uparrow$ Inflammation & $\uparrow$ Нypoxia & $\begin{array}{l}\uparrow \text { Immune } \\
\text { activation }\end{array}$ & $\downarrow \mathbf{M H C}$ & Mutations & $\begin{array}{c}\text { Immune evasion \& } \\
\text { tolerance }\end{array}$ & $\begin{array}{l}\text { Oxidative stress, inflammation and immune } \\
\text { activation as therapeutic targets }\end{array}$ \\
\hline PubMed & *224 & 6,595 & 12,925 & 68,177 & 4424 & *697 & 220,071 & *188 & $* 5$ \\
\hline Google Scholar & $2,080,000$ & 955,000 & $3,140,000$ & $1,260,000$ & $2,910,000$ & 58,300 & $3,270,000$ & 38,100 & 139,100 \\
\hline ScienceDirect & 85,169 & 75,531 & 181,344 & 52,755 & 126,315 & 8,065 & 271,724 & 1,993 & $* 589$ \\
\hline Total & $2,165,393$ & $1,037,126$ & 3,334269 & $1,380,932$ & $3,040,739$ & 67,062 & 3,761,795 & 40,281 & 139,694 \\
\hline
\end{tabular}

Table 2. Existing studies related to important similarities between asymptomatic HIV infection and cancer

\begin{tabular}{|c|c|c|c|c|c|c|c|c|c|}
\hline $\begin{array}{c}\text { Databases/Asym } \\
\text { ptomatic HIV } \\
\text { infection }\end{array}$ & $\begin{array}{c}\uparrow \text { Oxidative } \\
\text { stress }\end{array}$ & $\downarrow$ Antioxidant & $\uparrow$ Inflammation & $\uparrow$ Hypoxia & $\begin{array}{l}\uparrow \text { Immune } \\
\text { activation }\end{array}$ & $\downarrow \mathbf{M H C}$ & Mutations & $\begin{array}{c}\text { Immune } \\
\text { evasion \& } \\
\text { tolerance }\end{array}$ & $\begin{array}{c}\text { Oxidative stress, } \\
\text { inflammation and } \\
\text { Immune activation } \\
\text { as therapeutic } \\
\text { targets }\end{array}$ \\
\hline PubMed & $* 2$ & $* 2$ & *35 & $* 0$ & *53 & $* 1$ & *162 & $* 1$ & $*_{0}$ \\
\hline Google Scholar & 19,600 & 13,300 & 81,400 & 18,600 & 79,800 & 6360 & 49,900 & $* 5,630$ & 10,900 \\
\hline Science Direct & 1,822 & 1334 & 9605 & 2012 & 7647 & $* 751$ & 7,333 & $* 280$ & *751 \\
\hline Total & 21,424 & 14,636 & 91,040 & 20,612 & 87,500 & 7112 & 57,395 & 5911 & 11,651 \\
\hline
\end{tabular}


Table 3. Existing studies relating to important differences between cancer and asymptomatic HIV infection

\begin{tabular}{|c|c|c|c|}
\hline Studies common differences/ Cancer & Metabolic reprogramming & Apoptosis resistance & Clonal selection \\
\hline PubMed & $* 1053$ & 24127 & $* 1514$ \\
\hline Google scholar & 57400 & 19400 & 236100 \\
\hline Science Direct & $* 5334$ & 77319 & 20691 \\
\hline Total & $\mathbf{6 3 , 7 8 7}$ & $\mathbf{1 2 0 , 8 4 6}$ & $\mathbf{2 5 8 , 3 0 5}$ \\
\hline
\end{tabular}

Table 4. Existing studies relating to important differences between asymptomatic HIV infection and cancer

\begin{tabular}{|c|c|c|c|}
\hline Studies common differences/ Asymptomatic HIV Infection & $\uparrow$ Apoptosis & Respiratory burst & Senescence \\
\hline PubMed & 47 & $* 4$ & $* 38$ \\
\hline Google scholar & 32,300 & 17800 & 5320 \\
\hline Science Direct & 3927 & $* 947$ & $* 494$ \\
\hline Total & $\mathbf{3 6 , 2 7 4}$ & $\mathbf{1 8 , 7 5 1}$ & $\mathbf{5 8 5 2}$ \\
\hline
\end{tabular}

\section{Results}

Table 1 summarizes existing studies on identified important similarities in cancer and asymptomatic HIV infection. Although, cancer, oxidative stress, mutations, increased inflammation, hypoxia, immune activation are widely studied, however, oxidative stress, inflammation, immune activation as therapeutic target, immune evasion and tolerance field is not as widely studied presenting a fertile ground for future research. * indicates not adequately published area.

Table 2 summarizes existing studies on identified important similarities in asymptomatic HIV infection and Cancer. Asymptomatic HIV infection, oxidative stress, antioxidants, inflammation, hypoxia, immune activation, mutations area is widely studied. However, asymptomatic HIV infection in relation to, MHC, immune evasion, tolerance, oxidative stress, inflammation and immune activation as therapeutic targets is not as widely studied providing a fertile ground for future research. * indicates not adequately published area.

Table 3 summarizes existing studies related to cancer and asymptomatic HIV infection as downloaded using the different databases. The studies relates to cancer, metabolic reprogramming, apoptosis, clonal selection, which are important differences in cancer and asymptomatic HIV infection, processes and mechanisms. Cancer, metabolic reprogramming, apoptosis resistance (resistance to cell death) and clonal selection field is widely studied. * Indicates not adequately published area.

Table 4 summarizes existing studies related to asymptomatic HIV infection and cancer as downloaded using the different databases. Apoptosis in asymptomatic HIV infection is well studied; however, respiratory burst and senescence are exhaustively studied providing and ideal area for future studies. * indicates areas not adequately published.

\section{Discussion}

Based on the search words; oxidative stress, cancer, asymptomatic HIV infection, chronic inflammation and immune activation, 15,284,572 from the total hits related to similarities between asymptomatic HIV infection and cancer were obtained. Only 503, 815 hits relating to the differences between the two diseases were obtained and after critical appraisal to assess their quality, methods, what was analyzed in the studies, results and relevance to the survey, 89 articles were selected. The results of the selected studies were compared and presented in form of summary tables as shown above.

Although oxidative stress, mutations, increased inflammation, hypoxia, immune activation in relation to cancer were widely studied according to the findings of this study, however, studies on oxidative stress, inflammation, immune activation, immune evasion and tolerance as therapeutic target were few, making these areas ideal targets for future research. Specifically, future research should focus on targeting signaling pathways involved in oxidative stress-induced inflammation, immune activation, how tumour cells evades the host's immune system or induces tolerance. Precisely, the role of oxidative stress and or ROS if at all, in rendering CD8-T lymphocytes dysfunctional, down regulation of $\mathrm{MHC} \mathrm{I}$ on their surfaces as well as a possible role in induction of the immunosuppressive T-regs present in both cancer and asymptomatic HIV infection should be delineated. This might provide novel, ideal future therapeutic targets and preventive strategies.

Notably, apoptosis in asymptomatic HIV infection is well studied; however, respiratory burst and senescence are not exhaustively investigated. This provides a perfect area in which future studies can focus their attention. Precisely, studies on the role of oxidative stress and or ROS in increased respiratory burst in asymptomatic HIV infection 
are required. Likewise, their role in the massive CD4 T-cells death, increased HIV replication, expression, dissemination and induction of "escape mutations", HIV variants among other survival adaptations the virus acquires during this phase should be delineated. Mburu et al. (2013) [28], albeit in a pilot study, were able to show increased respiratory burst in asymptomatic HIV infection and its contribution to the death of CD4 T-lymphocytes during this stage of this infection.

According to previous studies, increased and persistent ROS, oxidative stress-induced inflammation and immune activation are thought to cause sustained NF-kB and Ras signaling. Furthermore, increased ROS attack, oxidative stress, increased energy demands, competition for inadequate nutrients, oxygen by the rapidly dividing cells (thought to cause metabolic reprogramming and glycolytic influx), hypoxia, DNA damage, defects in cell cycle check points and dysfunctional telomere are believed to provide the selective pressure, which causes the cells to switch from apoptosis to resistance to cell death and undergo clonal selection as a survival adaptation for the harsh environment $[71,72]$. Subsequently, cells acquiring resistance to apoptosis are frequently more difficult to treat as they are also resistant to chemotherapies which primarily function by inducing apoptosis. For that reason, future therapeutic strategies should be designed with this resistance to cell death knowledge and ways of reversing, eliminating it or promoting apoptosis in mind [73].

The absence of rapidly dividing cells in asymptomatic HIV infection, hence no competition for inadequate resources but presence of the persistent oxidative stress attack, inflammation and immune activation, eliminates need for the cell to adapt or switch to resistance to apoptosis or cell death. However the ROS attack, resultant oxidative damage, viral load, infection, viral proteins and persistent NF-kB signaling are enough to activate apoptotic signaling in the CD4-T-cells to undergo apoptosis. Metabolic reprogramming in cancer is thought to be caused by the increased energy demands from the rapidly dividing cancer cells, oxidative stress, while the HIV infection and translocation of microbial products (LPS) from the gut to systemic circulation as a result of loss of integrity of gut mucosa (leaky gut) in asymptomatic HIV infection is believed to cause the increased respiratory burst $[74,75]$.

According to previous studies and findings of this study, senescence or activation of telomerase in carcinogenesis was probably an adaptations mechanisms by the rapidly dividing cells to compete favorably for the scarce nutrient resources and survive the persistent ROS or oxidative stress attack, which is absent in asymptomatic HIV infection [76]. Activation of telomerase activity by ROS and other carcinogens is believed to lead to senescence in cancer, but is absent in HIV infection [77]. Since the highly metabolizing, rapidly dividing cells requires new blood vessels to serve the new cells, the ensuing oxidative stress, hypoxia are important in induction of angiogenesis via induction of hypoxia-inducible factor I (HIF-I) and vascular endothelial growth factor (VEGF) [78].

Likewise, competition for inadequate nutrients, oxygen and cellular adaptations by the rapidly dividing cells which is present in cancer but absent in asymptomatic HIV infections could explain clonal selection present in cancer but absent in HIV [79]. While viral infection, translocation of microbial products from the gut to systemic circulation as a result of loss of integrity of gut mucosa causes oxidative stress, inflammation and immune activation in asymptomatic HIV infection: ROS, intrinsic oxidative stress, chemical carcinogens, cancer causing infectious bacteria, viruses and resultant tumour associated inflammation (TAI) are the source in cancer [10]. In both cancer and HIV/AIDS, immune activation, complement system activation, impairing of the immune system, CD8 T-cells damage are common features. In addition, increased generation of several cytokines, chemokines (interferons \& pro-inflammatory cytokines), growth factors, decreased antioxidant defenses, down regulation of MHC molecules and induction of the immunosuppressive T-regs are characteristic of the two diseases. Importantly, the integrity of the immune system determines the rate of progression of HIV to AIDS, prevention, recovery in cancer and is a risk factor for non-Hodgkin lymphomas [31]. Further, anti-apoptotic genes such as Bcl-2, Bcl-X are upregulated in cancer but downregulated in HIV, while pro-apoptotic genes e.g. Bax, Bak, Bim, Bad are down regulated in cancer and upregulated in HIV. Interestingly, whereas excess oxidative stress, inflammation, immune activation leads to apoptosis of CD4 T-cells, in asymptomatic HIV infection, they are believed to cause resistance to apoptosis or replicative immortality cancer $[48,76,78]$.

According to current literature, several factors were implicated in the shift from apoptotic signaling to replicative immortality or resistance to apoptosis such as; chronic cytokine activation from failed resolution of tumour associated inflammation [77, 78, 79], as an adaptation to oxidative stress, decreased antioxidant defenses, competition for scarce resources nutrients, oxygen by the rapidly dividing cells, persistent attack from ROS, ensuing hypoxia, inhibition of caspases due to decreased glutathione or glucose influx, activation of telomerase by ROS, oxidative stress-induced metabolic dysregulation. This is believed to activate or cause "driver mutations" in proto-oncogenes (Ras \& c-Myc), inducing mutations and inactivating tumour suppressor genes (p153 \& Rb) [74, 79]. Importantly, absence of this competition for nutrients and oxygen in HIV/AIDS eliminates the need for the cellular adaptations. However, the persistent ROS, oxidative stress and inflammation still impacts on cells leading to apoptosis of the infected and affected CD4 T-lymphocytes. The increased catabolic and anabolic demands from the rapidly dividing cells which is absent in asymptomatic HIV infection, increased competition for nutrients, ROS, oxidative stress are thought to be the drivers of metabolic reprogramming characteristic 
of carcinogenesis. Significantly, clonal selection arises from the competition for scarce resources from the rapidly dividing cancer cells. The drivers of oxidative stress, inflammation and immune activation in cancer are mainly the infectious agents (cancer-causing viruses \& bacteria), exposure to carcinogens such as irradiation, ROS or environmental chemicals [22, 23], while the virus and translocation of microbial products from the gut mucosal to systemic circulation are the drivers in HIV infection [46]. Notably, anti-apoptotic genes are upregulated in cancer whereas pro-apoptotic genes are down regulated. The opposite is true in asymptomatic HIV infection [79]. Since cancer is multifactorial in aetiology involving many pathways, processes, a multiprocess and multitarget therapeutic approach is recommended as opposed to single enzyme and or pathway targeting [75].

\section{Identified Knowledge, Research Gaps and Future Research Direction}

Despite cancer and HIV being non-communicable disease and infectious diseases respectively, striking similarities in their pathophysiology are well recognized. These similarities include; persistent NF-kB signaling, altered metabolism (glycolytic influx \& metabolic reprogramming) in cancer versus increased respiratory burst in HIV. Similarly, HIV is caused by a virus while several virus-inducing cancers such as Epstein Barr Virus (EBV), Human papilloma virus (HPV), Hepatitis B \& C and emerging HIV associated malignancies) exists. Significantly, development of mutations such as “driver mutations” of proto-oncogenes (Ras \& Myc) and tumour suppressor genes (p53 \& Rb) in cancer [81], "escape mutations" in HIV [82,83], induction of immunosuppressive T-regs, immunosuppression and decreased immunosurveillance role in both (e.g. increased risk of cancer development in HIV-infected patients and organ transplant recipients) have been reported. Further, residual cancer stem cells (CSC) in cancer versus residual latent HIV infected CD4 T-cells, increased replication of the virus and its dissemination versus rapidly dividing cell in cancer, metastasis, invasion of tumour cells, decreased antioxidant defenses in both, dysfunctional CD8 T-lymphocytes by down regulation of MHC 1 molecules on CD8 T-cells in both diseases [30, 31], have been highlighted in previous studies. Significantly, chronic oxidative stress, inflammation and immune activation have been reported to be common in both the diseases [32, 34, 35]. Therefore, the author hypothesizes that these similarities are correspondingly initiated, driven and the "common driver" is believed to be the chronic oxidative stress, inflammation and immune activation, which characterizes the two diseases. Interestingly, oxidative stress has been recognized as the "master key" through which tumour microenvironment interacts with almost all cancer hallmarks [12].

However, in addition to the similarities, characteristic differences between the two diseases exist. These include
CD4 T-cells being the primary targets in HIV infection while several cell lineages can be targeted in cancers, clonal selection occurring only in cancers but absent in HIV infection. Significantly, apoptosis of CD4 T-lymphocytes occurs in HIV infection leading to massive death of the cells as opposed to resistance to apoptosis or cell death, sustained proliferative signaling, replicative immortality of cancer cells, leading to uncontrollable cell division and development of tumours in cancer. Senescence (aging) of T-cells as a result of thymus burn out, which is present in advanced HIV infection, is absent in cancer. Interestingly, cancerous cells do not senescence and have increased telomerase activity, attributed to ROS attack, persistent oxidative stress and which are thought to also contribute to metabolic reprogramming characteristic in cancers. Of specific importance, despite the stated similarities, HIV/AIDS is caused by a virus (HIV), while cancer has a multifactorial etiology that includes viruses, bacteria, chemical, environmental carcinogens and ionization [84, 85, $86,87]$.

Understanding the common drivers or pathophysiology of these similarities and differences can be applied to understand better carcinogenesis and its progression. Evidence from previous studies analyzed confirms presence of chronic oxidative stress, inflammation and immune activation in the two diseases making the author to hypothesize they are the "common drivers" of the similarities in pathophysiology of the two diseases. However, he recommends further studies specifically focusing on the role and impact of the same in the two diseases specially focusing on carcinogenesis to support and further strengthen this hypothesis.

\section{Future Challenges}

Although, the areas of cancer and asymptomatic HIV infection are well studied independently (as show in Tables 1-4) and important similarities, differences between the two documented, currently, no studies have ever linked the similarities and differences between the two, thus missing the opportunity to exploit this knowledge for better understanding of the two diseases. Despite, previous studies clearly highlighting some common similarities shared by cancer and asymptomatic HIV infection, this important knowledge has never been applied to understand better carcinogenesis, tumour maintenance and progression, which could accelerate development of therapeutic targets and preventive strategies. The rate at which cancer is killing people especially in resource limited settings such as Kenya, does not give us any laxity to miss such a massive opportunity, these similarities and differences between the two diseases offer. Of major importance as discussed in the introduction and indicated by the results in Table 1, 2, the numerous similarities in pathophysiology shared by the two diseases suggest a common driver. This evidence emboldens the author's believe that chronic oxidative stress, 
inflammation and immune activation, which characterizes the two diseases are the "drivers" of the similarities identified. Importantly, and in support of this hypothesis, inflammation, immune activation have been recognized as stronger predictors of HIV pathogenesis than CD4 T-cells count and viral load $[65,70]$. This highlights the important role oxidative stress, inflammation and immune activation plays in apoptosis of CD4 T-cells in asymptomatic HIV infection. As a result, this knowledge or understanding of oxidative stress-induced inflammation, immune activation mechanisms, processes in the two diseases hold the key to better understanding of carcinogenesis. Importantly, targeting signaling pathways involved in activation of these processes might provide ideal and novel therapeutic targets. Besides, modulation of oxidative stress-induced inflammation and immune activation using selected antioxidants had been shown to protect or inhibit CD4 T-cell death in asymptomatic HIV infection [8]. In support of this, $N$-acetyl cysteine (NAC), a vital thiol antioxidant, has been shown to influence telomerase activity, inhibiting senescence in cancer cells [88]. Likewise, the daily use of small doses of aspirin and other non-steroidal anti-inflammatory drugs (NSAIDs) was shown to decrease cancer risk [89], implying that oxidative stress, inflammation and immune activation are active participants in carcinogenesis.

This therefore demands a paradigm shift in our thinking, to start appreciating more the role played by these "drivers", focus more future research on them, their specific role or as cancer therapeutic targets and preventive strategies. Previously, oxidative stress, inflammation and immune activation were thought of as consequences but not causes of cancer. However, results of this descriptive review, supported by several earlier studies strongly suggest a causal association. Therefore, future studies, both in vitro, retrospective case-control studies focusing specifically on causal association of oxidative stress, inflammation, immune activation and cancer, their mechanistic actions, how to target their activation, signaling, modulation as therapeutical targets and preventive strategies are urgently required. The author concurs with previous authors [76], that some of the cancer hallmarks such as resistance to cell death (apoptosis), replicative immortality, metabolic reprogramming and clonal selection could probably be cellular adaptations to chronic oxidative stress.

As a recommendation, the role of ROS, oxidative stress-induce inflammation and immune activation in induction of metabolic reprogramming, persistent signaling of NF-kB, Ras, c-Myc, p53, Rb, anti-apoptotic gene Bcl-2, DNA damage, resistance to cell death, induction of "driver mutations" in proto-oncogenes and inactivation of tumour suppressor genes need to be exhaustively investigated. Notably, majority of the current cancer chemotherapies are limited in their action and ineffectiveness as they target single pathway, enzyme, indiscriminately kill tumour as well as normal cells, hence the many and serious adverse reaction associated with them. Therefore, the main future challenge, will be designing a safe, potent combination or a "magic bullet" capable of discriminating between cancers, normal cells, kill the cancer cells, working on several targets, signaling pathways, receptors and an efficient delivery system of the therapeutic formulations. A limitation of this study was that only three databases that were searched due to inaccessibility of others such as Medline and Scopus as a result of lack of subscription.

\section{Conclusions}

Cancer and asymptomatic HIV infection share important similarities and differences. Understanding of what drives these specific but essential similarities and differences in the two diseases might provide new mechanistic insights in carcinogenesis, provide future therapeutic targets, inform policy on cancer prevention, screening and early diagnosis. However, more studies including in vitro studies, retrospective case-control studies, focusing specifically on the role of these key drivers in carcinogenesis, cancer maintenance and progression are required to strengthen the hypothesis. In addition, information from this descriptive review will help in stimulating more research interest in the field, accelerate discovery of future novel therapeutic targets and preventive strategies. This will have a direct impact on cancer treatment, management and prevention. This will helping in attainment of the United Nations SDG number 3 on ensuring healthy lives and promotion of wellbeing for all at all ages and most importantly Kenya's vision 2030.

\section{Conflict of Interest}

The author declares no conflict of interest in conducting and publishing this review.

\section{REFERENCES}

[1] L. Torre. et al. Global Cancer Statistics. Cancer J. Clin, 65: 87-108. 2012.

[2] Joint United Nations Programme on HIV/AIDS (UNAIDS) Global Report. UNAIDS Report on the Global AIDS Epidemic 2013.

Geneva, Switzerland: UNAIDS; 2013. http:/files.unaids.org/ en/media/unaids/contentassets/documents/epidemiology/2013 /gr2013/UNAIDS_Global_Report_2013_en.pdf. downloaded on $25^{\text {th }}$ October, 2016

[3] P., Malloy, J., Boit, J. M., Allison Tarus, B., Ferrell \& Z. Ali. Providing palliative care to patients with cancer: Addressing the needs in Kenya. Asia-Pacific Journal of Oncology Nursing, 4(1), 45. , 2017.

[4] D.O., Kimanga, S., Ogola, \& M. Umuro. Prevalence and incidence of HIV infection, trends, and risk factors among 
persons aged 15-64 years in Kenya: results from a nationally representative study. Journal of acquired immune deficiency syndromes, (1999), 66(Suppl. 1), S13. (2014).

[5] WHO. Preventing Chronic Disease. A vital investment. [http:/ /www.who.int/healthinfo/global_burden_disease/2004_report _update/en/](2004). Downloaded on $20^{\text {th }}$ October, 2016.

[6] S. Franceschi. et al: Changing patterns of cancer incidence in the early-and late-HAART periods: The Swiss HIV Cohort Study. Br J Cancer, 103:416-422, 2010.

[7] K, Degenhardt et al. Autophagy promotes tumor cell survival and restricts necrosis, inflammation, and tumorigenesis. Cancer Cell. 10(1):51-64. 2006.

[8] S., Mburu, J. L., Marnewick, A., Abayomi, H. Ipp. Modulation of LPS- induced T-cell activation by antioxidants in asymptomatic HIV infection. Clinical \& Developmental Immunology, Vol. 2013, ID 631063, 2013.

[9] R. Truhaut. Recent progress in the evaluation of the dangers of chemical carcinogenesis. J. Pharm. Belg, 45, 131-140. 1990.

[10] S. Shishoda. et al. Curcumin (diferuloymethane) downregulates cigarette smoke-induced NF-kB activation through inhibition of IkappaBalpha kinase in human lung epithelial cells: correlation with suppression of COX-2, MMP-9 and cyclin D1. Carcinogenesis, 24, 1269-1279. 2003.

[11] S.K. Srivastava. et al. Cell cycle arrest, apoptosis induction and inhibition of nuclear factor kappa B activation in anti-proliferative activity of benzyl isothiocyanate against human pancreatic cancer cells. Carcinogenesis, 25, 1701-1709. 2004.

[12] A., Conti, C., Gulì, D., La Torre, C., Tomasello, F. F., Angileri, \& M. H. Aguennouz. Role of inflammation and oxidative stress mediators in gliomas. Cancers, 2(2), 693-712. 2010.

[13] S.C. Casey. et al. The effects of environmental chemicals on tumour microenvironment. Carcinogenesis, 36, S160-S183. 2015.

[14] T. Fiaschi. et al. Oxidative stress, tumour microenvironment and metabolic reprogramming, a diabolical liason. Int. J. Cell Biol., 2012, 762825. 2012.

[15] Trachootham. et al. Targeting Cancer cells by ROS-mediated mechanisms. A radical therapeutic approach? Nature Reviews, 8: 579-591. 2009.

[16] S. J., Ralph, S., Rodríguez-Enríquez, J., Neuzil, E., Saavedra \& R. Moreno-Sánchez. The causes of cancer revisited: "mitochondrial malignancy" and ROS-induced oncogenic transformation-why mitochondria are targets for cancer therapy. Molecular Aspects of Medicine, 31(2), 145-170. 2010.

[17] Y., Mitsuishi, K., Taguchi, Y., Kawatani, T., Shibata, T., Nukiwa, H. Aburatani \& H. Motohashi. Nrf2 redirects glucose and glutamine into anabolic pathways in metabolic reprogramming. Cancer Cell, 22(1), 66-79. 2012.

[18] S., Reuter, S. C., Gupta, M. M., Chaturvedi, \& B. B. Aggarwal. Oxidative stress, inflammation, and cancer: how are they linked?. Free Radical Biology and Medicine, 49(11), 1603-1616. 2010.

[19] D. J., Kurz S., Y., Decary, E., Hong, A., Trivier, J. Akhmedov \& D. Erusalimsky. Chronic oxidative stress compromises telomere integrity and accelerates the onset of senescence in human endothelial cells. Journal of Cell Science, 117(11), 2417-2426. 2004.

[20] L.B. Meira. et al. DNA damage induced by chronic inflammation contributes to colon carcinogenesis in mice. $J$. Clin. Invest., 118, 2516-2525. 2008.

[21] H.C. Hasselbalch. Chronic inflammation as a promotor of mutagenesis in essential thrombocythemia, polycythemia Vera and myelofibrosis. A human inflammation model for cancer development? Leuk. Res., 37, 214-220. 2013.

[22] V. Maguer-Satta. The stem cell niche: the black master of cancer, Cancer Stem Cells Theories and Practices, ISBN 978-953-307-225-8. 2011.

[23] S. Laconi. et al. A growth constrained environment drives tumour progression in vivo. Proc. Natl Acad. Sci. USA, 98, 7806-7811. 2001.

[24] G. Baffy, et al. Mitochondrial recoupling: a novel therapeutic strategy for cancer? Br. J. Cancer, 105, 469-474. (2011).

[25] M. L. Ryder. et al. Elevated phagocytosis, oxidative burst and F-actin formation in PMN'S from individuals with intra oral manifestations of HIV infection. Journal of AIDS, 1: 346-355. 1986.

[26] F. Aquaro, et al. Oxidative stress and HIV infection: Target pathways for novel therapies. Future HIV Therapy, 2: 327-338. 2008.

[27] C. Michailidis. et al. Impaired phagocytosis among patients infected by the human immunodeficiency virus: Implications for a role of highly active anti-retroviral. Clinical \& Experimental Immunology, 167: 3. 2012.

[28] S., Mburu, J.L., Marnewick, A., Abayomi, H. Ipp. Increased respiratory burst in asymptomatic HIV infection. A pilot study (Unpublished data).

[29] J.A. Knight. "Review: free radicals, antioxidants, and the immune system." Annals of Clinical \& Laboratory Science, 30.2 145-158. 2000.

[30] J. Kravchenko et al. Chemical compounds from anthropogenic environment and immune evasion mechanisms: potential interactions. Carcinogenesis, 36, 111-127. 2015.

[31] R.F. Ambinder. Infectious etiology of lymphoma. Williams and Wilkins, Philadelphia: 83-101. 2010.

[32] N.A. Karrow, et al. Oral exposure to atrazine modulates cell-mediated immune function and decreases host resistance to the B16F10 tumour model in female B6C3FF1 mice. Toxicology, 209, 15-28. 2005.

[33] L.M. Pinchuk, et al. In vitro atrazine exposure affects the phenotypic and functional maturation of dendritic cells. Toxicol. Appl. Pharmacol. 25, 206- 217. 2007.

[34] D. Hanahah. et al. The hallmarks of cancer, Cell, 100, 57-70. 2000.

[35] F. Colota. et al. Cancer-related inflammation, the seventh hallmark of cancer: link to genetic instability. Carcinogenesis, 30, 1073-1081. 2009.

[36] D. Hanahah. et al. Hallmarks of cancer: the next generation. Cell, 144, 646-674. 2011. 
[37] Pylayeva-Gupta, Yuliya, Elda Grabocka, and Dafna Bar-Sagi. "RAS oncogenes: weaving a tumorigenic web." Nature Reviews Cancer, 11.11. 761-774. 2011.

[38] Eric G. Wright. "Radiation-induced genomic instability and bystander effects: inter-related inflammatory-type non-targeted effects of exposure to ionizing radiation." Non-Targeted Effects of Ionising Radiation, 143. 2008.

[39] WHO. Consolidated guidelines on the use of antiretroviral drugs for treating and preventing HIV infection. Recommendations for a public health approach, 2013. http://apps.who.int/iris/bitstream/10665/85321/1/9789241505 727_eng.pdf downloaded on 10th September, 2016.

[40] V. Appay, \& D. Sauce, Handbook on Immunosenescence. Springer Science. Paris. 2009.

[41] J. C., Bundres, J., Trial, M. D., Musher, R. D. Rossen. Increased phagocytosis and generation of reactive oxygen products by neutrophils and monocytes of men with stage 1 HIV infection. Journal of Infectious Disease, 168: 75-83. 1993.

[42] A., Favier, C., Sappey, P., Leclerc, P. Faure, \& M. Micoud. Antioxidant status and lipid peroxidation in patients infected with HIV. Chemical Biology Interaction, 91: 165-180. 1994.

[43] C. Jarstrand \& B. Akerlund. Oxygen radical release by neutrophils of HIV-infected patients. Chemical Biological Interactions, 91: 141-146. 1994.

[44] A. Pugliese. et al. Phagocytic Activity in Human Immunodeficiency Virus Type 1 Infection. Clinical and Diagnostic Laboratory Immunology, 12: 889-895. 2005.

[45] E., Jacotot, L.,Ravagnan, M., Loeffler, K. F., Ferri, H. L., Vieira, N., Zamzami,... \& T. Irinopoulou. The HIV-1 viral protein $\mathrm{R}$ induces apoptosis via a direct effect on the mitochondrial permeability transition pore. Journal of Experimental Medicine, 191(1), 33-46.2000.

[46] J.M. Brenchley. et al. Microbial translocation is a cause of systemic immune activation in chronic HIV infection. Nature Medicine, 12: 1365-1371. 2006.

[47] R. Franco \& M. I. Panayiotidis. Environmental toxicity, oxidative stress, human disease and the" black box" of their synergism: how much have we revealed? Mutation Research, 674: 1-2. 2009.

[48] L., Gil, G., Martinez, I., Gonzalez, A., Tarinas, A. Alvarez, \& A. Guliana. Contribution to characterization of oxidative stress in HIV/AIDS patients. Pharmacological Research, 47: 217-224. 2003.

[49] P. Pasupathi, T. Ramachandran, P. J. Sindhu, G. Saravanan \& G. Bakthavathsalam. Enhanced oxidative stress markers and antioxidant imbalance in HIV infection and AIDS patients. Journal of Scientific Research, 1: 370-380. 2009.

[50] A. Wanchu, S.V. Rana, S. Pallikkuth, \& R. K. Sachdeva. Short Communication: Oxidative stress in HIV-infected individuals: A cross sectional study; 2009. Aids Research and Human Retroviruses, 25: 12. 2009.

[51] R. L. Prior \& G. Cao In vivo total antioxidant capacity: comparison of different analytical methods. Free Radical Biology and Medicine, 27: 1173-1181. 1999.

[52] T. P. A., Devasagayama, J. C., Tilak, K. K., Boloor, K. S.,
Sane, S. S. Ghaskadbi, \& R. D. Lele. Free radicals and antioxidants in Human health: Current status and future prospects. Journal Association of Physicians India, 52: 794-803. 2004.

[53] D. P. Kotler. Antioxidant therapy and HIV. The American Journal of Clinical Nutrition, 67: 5-9. 1998.

[54] L. G. Israels, \& E. D. Israels. Mechanisms in Haematology. Ontario, Core Health Services Inc. 2002.

[55] L. M., Prescott, J. O. Harley \& D. A. Klein. Microbiology. $5^{\text {th }}$ ed. New York: McGraw-Hill. 2002.

[56] E. P., Reeves, H., Lu, H. L., Jacobs, C. G., Messina, S., Bolsover, G. Gabella. \& A. W. Segal. Killing activity of neutrophils is mediated through activation of proteases by $\mathrm{K}+$ flux. Nature, 416: 291-97. 2002.

[57] B. F. Haynes. et al. Gut microbes out of control in HIV I infection. Nature Medicine, 12: 1365-1371. 2006.

[58] E. Nixon \& A. L. Landay. Biomarkers of immune dysfunction in HIV. Current Opinions in HIV and AIDS, 5: 498-503. 2010.

[59] M. D. Hazenberg. et al. Persistent immune activation in HIV-1infection is associated with progression to AIDS. AIDS, 17: 1881-1888. 2003.

[60] E. Cassol. et al. Persistent microbial translocation and immune activation in HIV 1-infected South Africans Receiving combined antiretroviral therapy. The Journal of Infectious Diseases. 5: 723-733. 2010.

[61] D. Morris. et al. Unveiling the mechanisms for decreased glutathione in individuals with HIV infection. Clinical and Developmental Immunology, ID 734125. 2012.

[62] G. W. Pace \& C. D. Leaf. The role of oxidative stress in HIV disease. Free Radical Biology \& Medicine, 19: 523-528. 1995.

[63] R., Tan, A. O., Westfall, J. H., Willig, M. J., Mugavero, M. S., Saag, R. A. Kaslow, \& M. C. Kempf. Clinical outcome of HIV-infected antiretroviral-naive patients with discordant immunologic and virologic responses to highly active antiretroviral therapy. Journal of Acquired Immune Deficiency Syndrome, 47: 553-558. 2008.

[64] P. C. Tien, et al. Inflammation and Mortality in HIV-Infected Adults: Analysis of the FRAM Study Cohort. Journal of Acquired Immune Deficiency Syndrome, 55:316-322. 2010.

[65] D. Nolan. et al. Host genetics and viral infections: immunology taught by viruses, virology taught by the immune system. Current Opinion in Immunology, 18.4 413-421. 2006.

[66] J. V., Giorgi L. E., Hultin J. A., McKeating T. D., Johnson, B., Owens, L. P. Jacobson. Shorter survival in advanced human immunodeficiency virus type 1 infection is more closely associated with $\mathrm{T}$ lymphocyte activation than with plasma virus burden or virus chemokine coreceptor usage. Journal of Infectious Disease, 179: 859-870. 1999.

[67] S. G. Deeks. et al. Immune activation set point during early HIV infection predicts subsequent CD4+ T-cell changes independent of viral load. Blood, 104: 942-947. 2004.

[68] A. Ashkenazi \& V. M. Dixit. Apoptosis control by death and decoy receptors. Current Opinion in Cell Biology, 11: 255-260. 1999.

[69] K. J., Tracey \& A. Cerami. Tumor necrosis factor, other 
cytokines and disease. Annual Review of Cell Biology, 9(1), 317-343.1993.

[70] Z. Liu. Molecular mechanism of TNF signaling and beyond. Cell Research, 15: 24-27. 2005.

[71] J. L., Fahey, J. G. M., Taylor, B., Manna, P., Nishanian, N., Aziz, J. V. Giorgi. Prognostic significance of plasma markers of immune activation, HIV viral load and CD4 T-cell measurements. AIDS, 12: 1581-1590. 1998.

[72] W. Bal. et al. Induction of oxidative DNA damage by carcinogenic metals. Toxicol. Lett., 127, 55-62. 2002.

[73] R.W. Johnstone. et al. Apoptosis: a link between cancer genetics and chemotherapy. Cell, 108, 153-164. 2002.

[74] S.W. Fesik. Promoting apoptosis as a strategy for cancer drug discovery. Nat. Rev. Cancer, 5, 876-885. 2005.

[75] R.J. DeBerardinis, et al. The biology of cancer: metabolic reprogramming fuels cell growth and proliferation. Cell Metab., 7, 11-20. 2008.

[76] R.B. Robey et al. Metabolic reprogramming and dysregulated metabolism: cause, consequence and/or enabler of environmental carcinogenesis?. Carcinogenesis, 36, S203-S231. 2015.

[77] M. Hosokawa. A higher oxidative status accelerates senescence and aggravates age-dependent disorders in SAMP strains of mice. Mech. Ageing Dev., 123, 1553-1561. 2002.

[78] F. Colotta. et al. Cancer-related inflammation, the seventh hallmark of cancer: links to genetic instability. Carcinogenesis, 30, 1073-1081. 2009.

[79] J. Candido, et al. Cancer-related inflammation. J. Clin. Immunol., 33 (suppl. 1), S79-S84. 2013.
[80] E.A. Grimm, et al. Molecular pathways: inflammation-associated nitric-oxide production as a cancer-supporting redox mechanism and a potential therapeutic target. Clin. Cancer Res., 19, 5557-5563. 2013.

[81] P. Cirri. et al. Cancer associated fibroblasts: the dark side of the coin. Am. J. Cancer Res., 1, 482-497. 2011.

[82] Y.L. Yang. et al. The IAP family: endogenous caspase inhibitors with multiple biological activities. Cell Res., 10, 169-177. 2000.

[83] A. D., Kelleher, C., Long, E. C., Holmes, R. L., Allen, J., Wilson, C., Conlon \& C. Brander. Clustered mutations in HIV-1 gag are consistently required for escape from HLA-B27-restricted cytotoxic $\mathrm{T}$ lymphocyte responses. Journal of Experimental Medicine, 193(3), 375-386. 2001.

[84] A. Carnero. et al. Disruptive chemicals, senescence and immortality. Carcinogenesis, 36, s19-S37. 2015.

[85] C.L. Boutwell. et al. Viral evolution and escape during acute HIV-1 infection. Journal of Infectious Diseases, 202 (Suppl. 2): S 309-14. 2010.

[86] L.M. Ferreira. et al. Metabolic reprogramming of the tumor. Oncogene, 31.36, 3999-4011. 2012.

[87] M.J. Gonzalez. et al. The bioenergetics theory of carcinogenesis. Med. Hypothesis, 79, 433-439. 2012.

[88] A.C. Lee. et al. Ras proteins induce senescence by altering the intracellular levels of reactive oxygen species. J. Biol. Chem., 274, 7936-7940. 1999

[89] E.M. Moran. Epidemiological and clinical aspects of non-steroidal anti-inflammatory drugs and cancer risks. $J$. Environ. Pathol. Toxicol. Oncol., 21, 193-201.2002. 